\title{
KANT Y EL PENSAMIENTO LIBERAL CONTEMPORÁNEO
}

Paulette Dieterlen

INSTTUTOO DE INVESTIGACIONES FILOSGFICAS Universidad Nacional Autónoma de MExico

Ronald Dworkin en un artículo llamado "Liberalism" 1 se propone la tarea de investigar qué elementos son constitutivos del conjunto de ideas llamado "liberalismo". Sin entrar en detalles sobre este antículo me referiré a algunos comunes denominadores que el mismo Dworkin discute. Generalmente asociamos liberalismo con la defensa de los derechos individuales, con el mercado libre, con la propiedad privada, con la democracia $y$, en fin, con los avatares que sufre el capitalista para poder invertir y de cuyos frutos se "beneficia" sin duda alguna la clase obrera. Los nombres que nos vienen a la mente cuando hablamos de liberalismo son el de John Stuart Mill, el de David Ricardo, incluso el del promotor de la economía del bienestar John Maynard Keynes.

El objeto de este trabajo es mostrar que dentro de las ideas liberales se encuentra el pensamiento kantiano; que su base moral no está dada por las doctrinas éticas de los mismos liberales, como sería el caso del utilitarismo de Mill, sino en las formulaciones del imperativo categórico de Kant.

Las preguntas que se nos antoja formular son: ¿Qué tiene que decirnos Kant, cuyos escritos políticos son secundarios en relación con sus tres "Críticas"? ¿Qué puede decir el maestro de Koenigsberg a un mundo tan agitado como en el que vivimos actualmente? Si su obra política no causó estragos en la Prusia de Federico II, ¿cómo es posible que sus ideas permanezcan vigentes?

Dos autores contemporáneos, portadores de una bandera liberal, se inspiran en Kant para elaborar sus teorias políticas. Caracterizaré la base kantiana de la Teoria de la justicia ${ }^{2}$ de John Rawls como una interpretación igualitaria del imperativo categórico, y la de Robert Nozick en Anarquia, Estado y Utopia ${ }^{3}$ como una interpretación anti-igualitaria

1 Dworkin R., "Liberalism", en Public and Private Morality, S. Hampshire Ed., Cambridge University Press, 1978, pp. 113-143.

2 Rawls, John, Teoria de la justicia, México, Fondo de Cultura Económica, 1979.

s Nozick, Robert, Anarchy, State and Utopia, Oxford, Blackwell, 1980. 
del mismo. Pero antes de pasar a examinar ambas interpretaciones hablemos un poco de Kant.

Es de todos sabido que la noción fundamental de la ética kantiana es la de imperativo categórico, noción que está intimamente ligada con la idea de actuar de acuerdo con el deber. Actúan de acuerdo con el deber aquellos seres que son racionales pero que tienen inclinaciones, las cuales son objeto de conflictos.

Usamos la palabra deber en distintos tipos de circunstancias, hay un uso propio de la moralidad y otros que no lo son. Por esta razón Kant distingue entre imperativos categóricos e imperativos hipotéticos, los que a su vez se subdividen en reglas de habilidades y consejos de prudencia.

Kant afirma que gran parte de nuestra educación depende de la adquisición de habilidades para hacer determinadas cosas. El uso que hagamos o no hagamos depende de nuestras elecciones y muchas veces esas elecciones son arbitrarias. Los imperativos propios de las reglas de habilidades son problemáticos porque los fines a los que se refieren son fines que no es necesario perseguir. Por ejemplo, si quiero ganar una maratón debo correr $50 \mathrm{~km}$. diarios, pero el fin, que en este caso sería el triunfo, puede ser sustituido por otro, como aprovechar la caminata para elaborar peripatéticamente un gran sistema filosófico. Kant denomina "técnicos" a estos imperativos."

Los consejos de prudencia son reglas que ayudan a los hombres a conseguir su felicidad. La diferencia entre éstos y los primeros es que los consejos de prudencia no son problemáticos pues todos queremos alcanzar la felicidad. Pero son asertóricos porque funcionan como simples medios para perseguir alguna finalidad. Por ejemplo, si quiero ser feliz debo superarme constantemente. Siendo el fin la felicidad, mi deber de superación queda subordinado a la categoría de medio. Estos son imperativos pragmáticos ${ }^{5}$ porque son aquellos en los que, según Kant, "la acción no es absolutamente ordenada sino que es medio para otros propósitos". ${ }^{6}$

Los deberes que son morales son los imperativos categóricos, cuya característica es la de ser leyes pues implican una concepción de una necesidad objetiva incondicional y en consecuencia son universalmente válidos.

Una persona que actúa de acuerdo con los principios del imperativo hipotético, actúa de una manera heterónoma; por el contrario, una per-

4 Kant, Fundamental Principles of the Metaphysic of Morals, Indianapolis, The Bobbs-Merrill Company, p. 34.

5 Ibid.

Ibid., p. 33. 
sona que actúa de acuerdo con el imperativo categórico, actúa autónomamente.

Podemos entender la conducta humana si descubrimos esas leyes y principios que son independientes de la experiencia y que Kant denomina como proposiciones prácticas sintéticas a priori. Estas proposiciones tienen la forma universal incondicional de los principios vacios de la lógica y el significado sustantivo no trivial de los principios de las matemáticas y de la física.

Para justificar el uso moral del deber, tenemos que suponer que el hombre no es sólo un ser fenomenal, sujeto a leyes causales estrictas, sino también un ser noumenal, es decir, libre y autónomo que tiene la capacidad de autolegislarse.

Podríamos preguntarnos acerca del criterio que necesitamos para saber si un deber es categórico y Kant nos da tres formulaciones, siendo la primera "cuando una máxima es susceptible de ser establecida como ley universal".

La segunda formulación está relacionada con la idea de que "los hombres no deben ser tratados solamente como medios sino siempre como fines". En virtud de este principio podemos saber qué acciones son correctas tanto en materia de moral como en política, ya que significa que no debemos usar a los demás ni a nosotros mismos como medios para lograr fines subjetivos.

La tercera formulación del imperativo categórico reza asi: "actúa de tal modo que seas, a través de tus máximas, un autolegislador, miembro del reino universal de los fines". Esta formulación implica una afinidad entre la moral y la política, puesto que "las acciones de los hombres no se llevan a cabo en el vacío, sino siempre con relación a otros hombres" y "esto sugiere implícitamente una teoría política, es decir, un sistema de principios que gobiernan las relaciones humanas organizadas".?

Las personas caracterizadas como agentes libres, racionales y autónomos establecen relaciones sociales y políticas que algunas veces suelen ser conflictivas, por esto se requiere de una resolución que sea universal y aquí surge la necesidad del orden legal. Mientras que las decisiones legales son "externas" y pueden ser forzadas, las decisiones morales son internas y no pueden ser ni forzadas ni reguladas. ${ }^{8}$

Kant formula el principio universal del derecho de la siguiente manera: "Es justa toda acción que por sí o por su máxima no es un obstáculo a la conformidad de la libertad del arbitrio de todos con la libertad de cada uno según leyes universales." 9

7 Reiss, Hans, "Introduction", en Kant's Political Writings, Cambridge University Press, 1970, p. 19.

8 Kant, Principios metafisicos de la doctrina del Derecho, Méxio, unAM 1978, p. 39.

I Ibid., p. 32. 
Los principios de la política son substancialmente los principios del derecho.

La investigación filosófica sobre la politica debe establecer qué acciones políticas son justas o injustas. Debe señalar por qué principios puede uno establecer las demandas de la justicia en una situación dada. La justicia debe ser universal y sólo el derecho puede garantizarla. Aś́ como en la ética de Kant deben estar basados en máximas capaces de ser formuladas como leyes universales, en polítca los acuerdos deberán estar organizados con base en leyes universalmente válidas.

De lo anteriormente expuesto podemos concluir que Kant parte del individuo para cuestionar la realidad política, ya que el principio del derecho es básicamente una aplicación del principio de la moralidad. No hay que olvidar que no busca un sistema - ya sea de moralidad, de derecho o de política - que dé cuenta de la diversidad empírica, sino una aproximación a dicho sistema elaborando aquellos principios $a$ priori y universales que considera relevantes.

Pasemos a descubrir brevemente la interpretación de Kant que he llamado igualitaria.

John Rawls en su famosa obra Teoría de la justicia afirma, al igual que Kant; que "la justicia es la virtud principal de las instituciones sociales", ${ }^{10}$ entendiendo la justicia como imparcialidad. Trata de exponer una doctrina en términos de un liberalismo igualitario cuya característica es el no ser perfeccionista ni mentocrático. Esto significa que no hay un modelo a seguir y que las personas no van a ocupar un lugar en la socièdad exclusivamente por su mérito. La justicia de las instituciones sociales tampoco puede ser medida por una tendencia a maximizar la suma o el promedio de ciertas ventajas, sino por la tendencia a contrarrestar las desigualdades naturales que se derivan del nacimiento y de los talentos.

Estas desigualdades pueden ser incorporadas al bien común, el cual se mide en términos de un conjunto restringido de beneficios para los individuos, como son la libertad personal y política, las ventajas sociales y económicas y el respeto propio.

La justicia de las instituciones depende de su conformidad con dos principios. El primero requiere la mayor libertad por igual, compatible con una libertad semejante para todos. El segundo principio, de la diferencia, requiere que sólo se permitan las desigualdades en la distribución de las ventajas económicas y sociales que benefician a todos pero principalmente a los menos favorecidos. Según Rawls se logra la imparcialidad si las personas pueden someterse a un procedimiento de elección para poder acordar sobre los principios que han de regular sus

10 Rawls, John, op. cit., p. 19. 
instituciones. La situación hipotética de la que se parte es la posición original.

La intención de la posición original es establecer un procedimiento equitativo según el cual cualesquiera que sean los principios convenidos, éstos sean justos.

Se trata, con esto, de anular los efectos de las contingencias especificas que ponen a los hombres en situaciones desiguales y en la tentación de explorar las circunstancias naturales y sociales en provecho de algunos.

Para lograr un acuerdo equitativo, Rawls supone que las partes están situadas bajo un "velo de ignorancia". No saben en qué medida las alternativas diversas afectarán sus casos particulares, viéndose asi obligados a evaluar los principios únicamente sobre la base de consideraciones generales. Las partes no conocen su lugar en la sociedad, su posición o clase social; tampoco saben cuál será su suerte una vez que se distribuyan los talentos y las capacidades naturales. Igualmente, nadie conoce ni su concepción del bien, ni los detalles de su plan racional de vida, ni siquiera los rasgos particulares de su propia psicologia. Más todavía, las partes no conocen las circunstancias particulares de su sociedad, esto es, no conocen su situación política o económica, ni el nivel de cultura y civilización que han sido capaces de alcanzar. Tampoco tienen ninguna información respecto a qué generación pertenecen.

Así pues, tendrán que escoger aquellos principios con cuyas consecuenciàs estén dispuestos a vivir, sean cuales sean las condiciones en las que se encuentren.

En la medida en que sea posible, el único hecho particular que conocen las partes es que su sociedad está sujeta a las circunstancias de la justicia. Se da por sentado que conocen los hechos generales acerca de la sociedad humana. Entienden las cuestiones políticas y los principios de la teoría económica, conocen las bases de la organización social y las leyes de la psicologia humana. "Se supone que conocen todos los hechos generales que afectan la elección de los principios de justicia." ${ }^{11}$

Rawls dice que su teoría moral está conectada con la filosofía de Kant particularmente por la interpretación que hace de la posición original. Afirma que la posición original puede ser considerada como una interpretación procesal de la concepción kantiana de la autonomfa y del imperativo categórico, dentro del sistema de una teoría empírica.

Podríamos decir que el velo de la ignorancia tiene el efecto de limitar a las partes en la posición original para que de esta manera no elijan principios que puedan ser particulares, problemáticos o asertóricos, es decir, principios hipotéticos. "Las personas llegan a una elección conjunta como personas racionales y libres que conocen únicamente aque-

11 Ibid., p. 164. 
llas circunstancias que dan lugar a la necesidad de los principios de justicia." 12

Si bien Rawls reconoce la influencia kantiana respecto a la situación original, también la reconoce respecto a las restricciones formales que es razonable imponer a las concepciones de justicia que se incluyen en la lista presentada a las partes. En primer lugar, los principios deberían ser generales. Esto es, tiene que ser posible formularlos sin el uso de palabras que intuitivamente podrían ser reconocidas como nombres propios o descripciones definidas: los primeros principios tienen que ser capaces de servir como base pública perpetua de una sociedad bien ordenada. En segundo lugar, los principios han de ser universales en su aplicación. Tienen que valer para todos por ser personas morales. Una tercera condición es la del carácter público, la cual surge del punto de vista contractualista. Las partes suponen que están escogiendo principios para una concepción pública de justicia. La publicidad es algo implícito en la noción que Kant tiene de la ley; el derecho público, afirma, "es la suma total de aquellas leyes que han de ser publicadas universalmente con el fin de producir un estado de derecho". ${ }^{13}$

Otra condición adicional es que una concepción de lo justo tiene que imponer una ordenación de las demandas conflictivas. La quinta y última condición es la de definitividad. No existen patrones más elevados a los que puedan remitirse los argumentos que apoyen demandas. Un razonamiento a partir de estos principios es concluyente.

Obviamente gran parte de la teoría de Rawls está destinada a probar que los únicos principios que cubren estos requisitos son los principios de la justicia que, como se mencionó antes, son: 1) La mayor libertad por igual, compatible con una libertad semejante para todos, y 2) sólo se permitirán aquellas desigualdades en la distribución de las ventajas económicas y sociales que beneficien a todos, pero principalmente a los menos favorecidos.

$\mathrm{La}$ interpretación que he denominado anti-igualitaria se encuentra en la obra Anarquia, Estado y Utopia. Robert Nozick parte de la idea de que los hombres tienen derechos, para concluir que el único tipo de Estado que no los viola es el Estado mínimo. Entiende por ello un Estado que solamente redistribuye protección; todas las demás funciones de distribución deben regirse por la ley de la oferta y la demanda.

Los derechos funcionan como "restricciones laterales morales" y no como metas que hay que conseguir. La diferencia entre estos puntos de vista es que de acuerdo con el último, alguien podría violar el derecho de otra persona si con ello se minimizara la suma total de viola-

12 Ibid., p. 289.

13 Kant, Principios metafisicos de la doctrina del Derecho, p. 139. 
ción de derechos. Si tomamos el primer punto de vista, el derecho de los otros determina las restricciones que se pueden hacer a las acciones.

De acuerdo con Nozick, las restricciones laterales reflejan la segunda formulación del imperativo categórico según la cual los individuos son fines y no solamente medios, "no pueden ser sacrificados o usados para lograr otros fines sin su consentimiento"; "los indivduos son inviolables". ${ }^{14}$ Esta idea nos lleva a formularnos unas preguntas: ¿por qué no podríamos violar a algunas personas si con ello logramos un bien social mayor? Nosotros mismos muy a menudo elegimos un dolor o un sacrificio para alcanzar un beneficio o para evitar un mal mayor, ¿no podríamos, en forma similar, sostener que algunas personas tendrían que soportar algunas cargas en función del bien común?

Nozick, afirmando la influencia de Kant, responde que "no existe una entidad social con una idea del bien. Sólo existen personas individuales, personas individuales y diferentes, con su propia vida individual". ${ }^{15}$

Las restricciones morales están basadas en el hecho de que los individuos poseen racionalidad, libre albedrío, agencia moral y tienen la capacidad de formarse el plan de vida que quieran llevar, esto es, tienen la capacidad de "darle un sentido a su vida".

De esta concepción de la inviolabilidad de los individuos, Nozick infiere que es injusto ponerle trabas a la libre empresa, que un sistema de impuestos viola los derechos de las personas y que cualquier modelo de distribución interfiere con la libertad.

Si bien Rawls, en su teoría de la justicia, considera el ejercicio de la libertad como el primer valor que debe ser garantizado, respetado y promovido por las instituciones para ser consideradas justas, no se olvida de la igualdad. Recordemos que al privar de información a las partes en la posición original, las coloca en una situación igualitaria. Asimismo, el segundo principio de justicia limita el alcance del primero, puesto que se admite que el ejercicio de la libertad tenga como resultado ciertas desigualdades, siempre y cuando éstas vayan en beneficio de los menos favorecidos.

Sin embargo, uno de los problemas que presenta la teoría de Rawls es la aceptación, carente de toda crítica, de ciertos presupuestos sociopolíticos y de los modelos de análisis que son propios de la teoría económica neo-clásica liberal. Podríamos detenernos a reflexionar sobre el uso que hace de ciertas palabras como racionalidad, autonomía, justicia, etcétera. Sin duda, aquí la historia tendría mucho que decirnos.

Nozick, por su parte, afirma que en su teoría no existe un presupuesto en favor de la igualdad: no se puede simplemente asumir que deba

14 Nozick, op. cit., p. 31.

15 Ibid., p. 33. 
ser parte de una teoría de la justicia. No se puede argumentar en favor de un Estado que interviene para remediar una situación en la que muchos tienen poco y pocos tienen mucho, si los últimos han adquirido lo que tienen justamente.

La segunda formulación del imperativo categórico de Kant, la máxima que nos lleva a tratar a las personas no solamente como medios sino como fines, en Nozick nos llevan a afirmar que todos los hombres deben ser tratados como fines pero que "algunos son más fines que otros".

Estas posiciones liberales nos plantean la necesidad de reflexionar acerca de dos problemas. El primero es de interpretación, que se hace patente cuando vemos las conclusiones tan distintas que se extraen del imperativo categórico. El segundo es de origen, porque Kant quizá nunca logró deducir fines objetivos y obligatorios del análisis de lo que es un agente racional. Quizá tampoco pudo establecer una validez universal incondicional de algún principio de la razón práctica. ${ }^{16}$

La razón de ello está en que tal vez no haya esa clase de principios.

16 Wolff, R. P., Understanding Rawls, Princeton University Press, 1977, p. 111. 